\title{
REHABILITACIÓN VESTIBULAR EN PACIENTES CON VÉRTIGO Y TRASTORNO DE LA PERSONALIDAD
}

\section{Vestibular rehabilitation in patients with vertigo and personality disorder}

\author{
Ana GUTIÉRREZ-GALLARDO; Carmen SALOM-COVEÑAS; Eulalia Carmen PORRAS-ALONSO \\ Hospital Universitario de Puerto Real. Servicio de Otorrinolaringología. Puerto Real. Cádiz. España \\ Correspondencia: ana_gutierrez15@hotmail.com
}

Fecha de recepción: 25 de marzo de 2021

Fecha de aceptación: 25 de mayo de 2021

Fecha de publicación: 27 de mayo de 2021

Fecha de publicación del fascículo: 1 de septiembre 2021

Conflicto de intereses: Los autores declaran no tener conflictos de intereses

Imágenes: Los autores declaran haber obtenido las imágenes con el permiso de los pacientes

Política de derechos y autoarchivo: se permite el autoarchivo de la versión post-print (SHERPA/RoMEO)

Licencia CC BY-NC-ND. Licencia Creative Commons Atribución-NoComercial-SinDerivar 4.0 Internacional

Universidad de Salamanca. Su comercialización está sujeta al permiso del editor

RESUMEN: Introducción y objetivo: La presencia de trastornos de la personalidad en pacientes con vértigo ha sido referenciada en diferentes trabajos. Las conexiones del sistema vestibular y el procesamiento de emociones es la causa que apoya dicha asociación. La rehabilitación vestibular se perfila como el tratamiento para el vértigo crónico no compensado, pero suscita la duda de si será suficiente por si sola en pacientes con trastornos de la personalidad o si debe acompañarse de otras terapias dirigidas a estas alteraciones. El objetivo del estudio es analizar la posible influencia de los trastornos de personalidad en la autopercepción de la discapacidad mediante el Dizziness Handicap Inventory (DHI) y en los parámetros de la posturografía estática, antes y después de la rehabilitación vestibular. Método: Estudio prospectivo de 55 pacientes diagnosticados de vértigo crónico periférico o central a los que se trató con ocho sesiones de rehabilitación vestibular. Los pacientes cumplimentaron el DHI y el cuestionario Salamanca al inicio y al final del tratamiento. Resultados: El 65\% de los pacientes tenían trastornos de la personalidad. Los rasgos de ansiedad se asociaron a vértigo periférico y los rasgos esquizoides a vértigo central. En todos los pacientes, la puntuación del DHI mejoró ( $\mathrm{p}<0,01$ ), al igual que algunos parámetros del control del limite de estabilidad $(\mathrm{p}<0.01)$. Por otro lado, no se encontró relación entre las variables independientes edad, sexo, diagnóstico clínico y trastorno de la personalidad con los resultados. Conclusiones: En nuestro estudio, los resultados 


\section{REHABILITACIÓN VESTIBULAR EN PACIENTES CON VÉRTIGO Y \\ TRASTORNO DE LA PERSONALIDAD \\ GUTIÉRREZ-GALLARDO A; SALOM-COVEÑAS C Y PORRAS-ALONSO E C}

muestran la eficacia de la rehabilitación vestibular en pacientes con vértigo crónico, independientemente de su asociación con trastornos de personalidad.

PALABRAS CLAVE: vértigo; trastornos de personalidad; rehabilitación vestibular.

SUMMARY: Introduction and objective: The presence of personality disorders in patients with vertigo has been reported in several articles. The connections between the vestibular system and emotion processing are the cause that supports said association. Vestibular rehabilitation is emerging as the treatment for chronic uncompensated vertigo, but it raises the question of whether it will be sufficient by itself in patients with personality disorders or whether it should be accompanied by other therapies aimed at these alterations. The aim of the study is to analyze the possible influence of personality disorders in the self-perception of disability using the Dizziness Handicap Inventory (DHI) and the parameters of static posturography, before and after vestibular rehabilitation. Method: Prospective study of 55 patients diagnosed with chronic vertigo treated with eight sessions of vestibular rehabilitation. The patients completed the DHI and the Salamanca questionnaire before and after the treatment was administered. Results: $65 \%$ of the patients had personality disorders. Anxiety traits were associated with peripheral vertigo and schizoid traits with central vertigo. In every patient, the DHI score improved $(\mathrm{p}<0,01)$, as well as some parameters of the control of the limit of stability $(\mathrm{p}<0,01)$. On the other hand, no relationship was found between the independent variables of age, sex, clinical diagnostic, personality disorder, and the results. Conclusions: In our study, the results show the efficacy of vestibular rehabilitation in patients with chronic vertigo, regardless of its association with personality disorders.

KEYWORDS: vertigo; personality disorders; vestibular rehabilitation.

\section{INTRODUCCIÓN}

El vértigo es una manifestación clínica de diferentes síndromes cuyo origen está en una disfunción vestibular y en ocasiones, en trastornos psiquiátricos o psicosomáticos. Sin embargo, la coexistencia de ambas patologías puede encontrarse hasta en un $30 \%$ de los pacientes estudiados [1]. Las alteraciones psiquiátricas a las que se asocia pueden diferir en función de la etiología del vértigo. En concreto, en la enfermedad de Ménière, el estrés psicológico relacionado con sus síntomas ha sido relacionado con niveles elevados de ansiedad y neuroticismo y en menor porcentaje, con cuadros depresivos.

En el tratamiento del vértigo y la inestabilidad crónica no compensada se ha propuesto la rehabilitación vestibular para mejorar la función del reflejo vestíbulo ocular, vestíbulo espinal así como la capacidad de habituación con otros sistemas. Se ha comprobado en diversos trabajos que existe una evidencia moderada sobre la efectividad de la rehabilitación vestibular en la mejora de los síntomas asociados a la disfunción vestibular periférica unilateral y en la percepción de la calidad de vida [2], disminuyendo su eficacia en pacientes con hipofunción vestibular bilateral y en mayores de 60 años.

En pacientes con trastornos de personalidad asociada existe cierta controversia de su eficacia como terapia única y se plantea la necesidad de incorporar una terapia cognitivo-conductual complementaria al tratamiento.

El objetivo de nuestro estudio es analizar la posible influencia de los trastornos de personalidad, establecidos por el cuestionario Salamanca $[3,4]$ en los resultados de la autopercepción de la discapacidad y su impacto en actividades de la vida diaria mediante el Dizziness Handicap Inventory (DHI) $[5,6]$ y en los resultados de la posturografía estática, antes y después de la rehabilitación vestibular. 


\section{MATERIAL Y MÉTODO}

Se diseñó un estudio prospectivo incluyendo 55 pacientes reclutados en un periodo de 18 meses con diagnóstico de vértigo sin mejoría clínica, clasificándose en patología vestibular periférica, central y no definida o mixta. En todos ellos, persistían síntomas tras seis meses desde el inicio del proceso.

Se excluyeron pacientes menores de edad, aquellos con dificultad física ó cognitiva para la realización de los ejercicios o que abandonaron el programa de rehabilitación sin completarlo.

Los criterios clínicos para clasificar a los pacientes de patología central fueron la presencia de síntomas vertiginosos menos intensos pero más prolongados en el tiempo que en el vértigo periférico, sin relación con la posición, inicio paulatino, sin acompañarse de hipoacusia ni síntomas vegetativos y además, lleven asociados otros síntomas neurológicos como diplopía o ataxia.

En lo que respecta a la exploración en el diagnóstico de un vértigo central, sin la necesidad de que estén todas las manifestaciones presentes, se encuentran: Romberg inestable o con caída variable, test del impulso cefálico negativo, nistagmo multidireccional o vertical que no se fatiga y no se inhibe con la fijación ocular, desviación ocular vertical a la realización de Cover test, alteración de la marcha, afectación de otros pares craneales, así como la presencia de dismetría en la prueba dedo-nariz. También se realizaron pruebas complementarias como análisis de sangre, RNM craneal y de poro acústico, así como punción lumbar si fuera necesario.

En algunos casos, la etiología resulta multifactorial dando lugar a un vértigo mixto o patología vestibular no definida.

En todos los pacientes se realizó exploración neurológica, audiometría tonal, impedanciometría y reflejo estapedial. Además, se incluyó valoración de nistagmo espontáneo con gafas de Frenzel, Head-Shaking test o agitación cefálica para evaluar el diagnóstico diferencial entre un nistagmus espontáneo fisiológico y patológico, tanto central como periférico, test de impulso cefálico de Halmagyi-Curthoys para determinar el reflejo vestíbulo ocular, test de Romberg y test de UnterbergerFukuda para valoración de la estática y la marcha, evaluación de nistagmo posicional mediante test de Dix-Hallpike y registro videonistagmográfico de pruebas calóricas Otopront ${ }^{\circledR}$ en ambos oídos. En función de la historia clínica y los resultados de la exploración se clasificaron en pacientes con vértigo periférico, central o no definido.

En la primera consulta los pacientes completaron el DHI, que cuantifica el impacto del vértigo en las actividades y situaciones propias de la vida diaria, englobando problemas específicos de orden funcional, emocional o físico, relacionados con trastornos del equilibrio; así como el cuestionario Salamanca, para valoración de trastornos de la personalidad.

En función de los resultados obtenidos del DHI, se clasificaron en pacientes con discapacidad leve de 0 a 30 puntos, discapacidad moderada de 30 a 60 puntos y discapacidad severa mayor de 60 puntos. Se consideró mejoría cuando se producía un cambio de discapacidad de severa a moderada, de moderada a leve o de severa a leve; también se calificó de mejoría una diferencia superior a 18 puntos entre el DHI inicial y final, independientemente de los grados de discapacidad.

El cuestionario Salamanca es un instrumento de cribado para evaluar los trastornos de personalidad con una alta sensibilidad y propiedades psicométricas que parecen corroborar su utilidad clínica, sobre todo en aquellos pacientes que presentan altas puntuaciones. Distribuye los trastornos de personalidad en tres grupos, grupo A con rasgos de personalidad paranoide, esquizoide y esquizotípico, grupo B con rasgos de personalidad histriónico, antisocial, narcisista, inestabilidad emocional subtipo impulsivo e inestabilidad emocional subtipo límite y grupo C con rasgos de personalidad anancástico, dependiente y ansioso. 


\section{REHABILITACIÓN VESTIBULAR EN PACIENTES CON VÉRTIGO Y \\ TRASTORNO DE LA PERSONALIDAD \\ GUTIÉRREZ-GALLARDO A; SALOM-COVEÑAS C Y PORRAS-ALONSO E C}

Las ocho sesiones de rehabilitación, dirigidas por enfermera especializada, se iniciaron con una primera fase informativa y firma de consentimiento informado. Posteriormente se ejecutaron ejercicios de un programa estándar dirigidos a la habituación vestibular, estabilización de la mirada, mejora del control vestíbulo ocular y postural, además de actividades para mejorar el estado general del paciente. Para ello, nos ayudamos de distintos elementos y materiales como rampas, pelotas, colchonetas y sillón giratorio. Además, en cada una de las sesiones también se realizaron ejercicios de entrenamiento en la plataforma Lucerne Otopront ${ }^{\circledR}$.

En la primera sesión los pacientes realizaron el test de Romberg con ojos abiertos, con ojos cerrados, con rotación de cabeza hacia la izquierda y rotación de cabeza hacia la derecha, así como Romberg intensificado. Se compararon los resultados del área de barrido $(\mathrm{cm} 2)$, velocidad media $(\mathrm{cm} / \mathrm{s})$, desplazamientos máximos laterales y anteroposterior $(\mathrm{cm})$ en cada una de los parámetros valorados al principio y al final de las sesiones.

De igual forma, para el estudio de los límites de estabilidad, los pacientes realizaban dos ejercicios de desplazamiento de su centro de gravedad sin cambios en la base de soporte en ocho dianas de diferente diámetro en un tiempo máximo de 90 segundos. Se compararon los resultados del área de barrido $(\mathrm{cm} 2)$, velocidad media $(\mathrm{cm} / \mathrm{s})$ y el recorrido máximo en su desplazamiento desde el centro hasta las dianas.

Por último, se comparó el porcentaje de relleno de una diana circular con desplazamiento de su centro de gravedad sin modificar la base de sustentación en un tiempo máximo de 60 segundos entre la primera y la última sesión.

\section{RESULTADOS}

La muestra incluyó 55 pacientes, 31 mujeres (56\%) y 25 hombres (44\%) con una edad media de 56 años y una desviación estándar de 11,42. Los pacientes con diagnóstico de disfunción vestibular periférica correspondieron a 36 casos $(65,5 \%)$, con un predominio de pacientes con síndrome de Ménière (47\%), disfunción vestibular central en 15 casos $(27,3 \%)$ y disfunción vestibular no definida a 4 casos $(7,2 \%)$.

El 65\% de la muestra, treinta y cinco pacientes, se asociaron a trastornos de personalidad entre los que destacan los rasgos esquizoides (26\%), histriónicos (26\%) y ansiosos (20\%). En el análisis de la relación entre los trastornos de personalidad y la diferente etiología del vértigo, encontramos un predominio de la ansiedad en el vértigo de origen periférico (30\%), correspondiendo en un 57\% de este grupo a pacientes diagnosticados de Síndrome de Ménière. Sin embargo, en el vértigo de origen central se observó un predominio de personalidad esquizoide alcanzando un porcentaje del $40 \%$ en este grupo (Tabla 1 ).

En la puntuación del DHI, si consideramos mejora en la discapacidad el cambio de discapacidad severa o moderada a discapacidad leve, ésta se observó en 35 pacientes, un $64 \%$ de la muestra. Si consideramos mejora en la discapacidad el cambio de discapacidad severa a moderada o de moderada a leve, ésta se observó en 45 pacientes, un $82 \%$ de la muestra. Si consideramos mejora la disminución en 18 puntos, ésta se observó en 48 pacientes, un $87 \%$ de la muestra.

Para valorar la diferencia de la puntuación del DHI y de resultados en los parámetros definidos previamente en cada uno de los test de la posturografía estática (área de barrido, velocidad media, desplazamientos laterales y anteroposterior, recorrido máximo en límites de estabilidad y porcentaje de cumplimiento en relleno de diana) al principio y final de la rehabilitación vestibular se aplicó el test de Wilcoxon no paramétrico para muestras pareadas.

Se obtuvieron diferencias estadísticamente significativas con una $\mathrm{p}<0,01$ en las puntuaciones del DHI inicial y final en cada una de las dimensiones (emocional, funcional y físico) para el total de la muestra y para cada uno de los subgrupos, pacientes 


\section{REHABILITACIÓN VESTIBULAR EN PACIENTES CON VÉRTIGO Y \\ TRASTORNO DE LA PERSONALIDAD \\ GUTIÉRREZ-GALLARDO A; SALOM-COVEÑAS C Y PORRAS-ALONSO E C}

Tabla 1. Pacientes con vértigo y trastornos de personalidad asociados.

\begin{tabular}{|l|c|c|c|}
\hline \multicolumn{1}{|c|}{ Trastornos de personalidad } & Vértigo Central & Vértigo Periférico & Otros \\
\hline GRUPO A & 4 & 5 & 1 \\
- Esquizoide & & 5 & \\
\hline GRUPO B & 3 & 1 & 1 \\
- Histriónico & & 1 & 1 \\
- Inestabilidad emocional impulsivo & 2 & 4 & 2 \\
- Inestabilidad emocional límite & 1 & 7 & \\
\hline GRUPO C & 10 & 23 & \\
- Anancástico & & & \\
- Ansioso & & & \\
- Dependiente & & & \\
\hline Número Pacientes $=35$ & & & \\
\hline
\end{tabular}

con trastornos de la personalidad y pacientes sin trastornos de la personalidad (Tabla 2).

Del mismo modo, se observaron diferencias significativas con una $\mathrm{p}<0,01$ en la velocidad media de los límites de estabilidad en los ejercicios de desplazamiento del centro de gravedad y en el porcentaje de relleno del círculo inicial y final, pero no en los diferentes parámetros del test de Romberg. Se analizaron los resultados en los grupos de paciente con trastornos de personalidad asociados, encontrando también diferencias estadísticamente significativas en los límites de estabilidad, aunque en las dianas de menor diámetro, la diferencia fue menor, con una $\mathrm{p}<0,05$ (Tabla 2).
Se estudió mediante el test de Chi Cuadrado la posible diferencia entre el comportamiento del DHI en el grupo de pacientes con trastorno de la personalidad y el grupo de pacientes sin trastorno de la personalidad, obteniéndose resultados que confirman un comportamiento similar en ambos grupos.

Asimismo, se evaluó mediante el test de Chi Cuadrado la posible diferencia entre la mejora objetiva del límite de estabilidad en el parámetro de velocidad media y el relleno del círculo entre el grupo de pacientes con trastorno de la personalidad y el grupo de pacientes sin trastorno, alcanzando igualmente resultados que confirman un comportamiento similar en ambos grupos.

Tabla 2. Parámetros con resultados significativos.

\begin{tabular}{|c|c|c|c|}
\hline & Inicial (medias) & Final (medias) & Estadístico \\
\hline $\begin{array}{l}\text { DHI todos los grupos } \\
\text { DHI grupo con trastorno de personalidad } \\
\text { DHI grupo sin trastorno de personalidad }\end{array}$ & $\begin{array}{c}68,44 \\
69,37 \\
66,8 \\
\end{array}$ & $\begin{array}{c}27,78 \\
25,66 \\
31,5\end{array}$ & $\begin{array}{l}\mathrm{p}<0,01 \\
\mathrm{p}<0,01 \\
\mathrm{p}<0,01\end{array}$ \\
\hline $\begin{array}{l}\text { LE. V. media } 1 \text { todos los grupos } \\
\text { LE. V. media } 1 \text { grupo con tras. personalidad } \\
\text { LE. V. media } 1 \text { grupo sin tras. personalidad }\end{array}$ & $\begin{array}{l}5,70 \mathrm{~cm} / \mathrm{s} \\
5,96 \mathrm{~cm} / \mathrm{s} \\
5,27 \mathrm{~cm} / \mathrm{s}\end{array}$ & $\begin{array}{l}7,46 \mathrm{~cm} / \mathrm{s} \\
7,79 \mathrm{~cm} / \mathrm{s} \\
6,87 \mathrm{~cm} / \mathrm{s}\end{array}$ & $\begin{array}{l}\mathrm{p}<0,01 \\
\mathrm{p}<0,01 \\
\mathrm{p}<0,01\end{array}$ \\
\hline $\begin{array}{l}\text { LE. V. media } 2 \text { todos los grupos } \\
\text { LE. V. media } 2 \text { grupo con tras. personalidad } \\
\text { LE. V. media } 2 \text { grupo sin tras. personalidad }\end{array}$ & $\begin{array}{l}4,71 \mathrm{~cm} / \mathrm{s} \\
4,98 \mathrm{~cm} / \mathrm{s} \\
4,25 \mathrm{~cm} / \mathrm{s}\end{array}$ & $\begin{array}{l}5,67 \mathrm{~cm} / \mathrm{s} \\
5,58 \mathrm{~cm} / \mathrm{s} \\
5,82 \mathrm{~cm} / \mathrm{s}\end{array}$ & $\begin{array}{l}\mathrm{p}<0,01 \\
\mathrm{p}<0,05 \\
\mathrm{p}<0,01\end{array}$ \\
\hline $\begin{array}{l}\text { LE. Círculo todos los grupos } \\
\text { LE. Círculo grupo con tras. personalidad } \\
\text { LE. Círculo grupo sin tras. personalidad }\end{array}$ & $\begin{array}{l}0,65 \\
0,65 \\
0,64\end{array}$ & $\begin{array}{l}0,78 \\
0,78 \\
0,78\end{array}$ & $\begin{array}{l}\mathrm{p}<0,01 \\
\mathrm{p}<0,01 \\
\mathrm{p}<0,01\end{array}$ \\
\hline
\end{tabular}

DHI $=$ Dizziness Handicap Inventory. LE $=$ Límites de estabilidad. V. media $=$ Velocidad media. Tras. Personalidad= trastorno de personalidad. 


\section{REHABILITACIÓN VESTIBULAR EN PACIENTES CON VÉRTIGO Y \\ TRASTORNO DE LA PERSONALIDAD \\ GUTIÉRREZ-GALLARDO A; SALOM-COVEÑAS C Y PORRAS-ALONSO E C}

Con el objetivo de establecer una posible relación entre la mejora de las puntuaciones del DHI final y alguna variable, se realizó un análisis de regresión logística considerando como variables independientes la edad, sexo, presencia de trastorno de personalidad y diagnóstico clínico. Como variable dependiente se definió la mejoría en los síntomas del paciente si la puntuación del DHI final disminuía en 18 puntos. No se identificó ninguna variable que estuviera relacionada con la mejora de forma significativa, si bien en la variable edad se obtuvo un valor de $\mathrm{p}<0,057$ cercano a la significación (Tabla 3).

Igualmente, se realizó análisis de regresión logística con las mismas variables independientes y como variables dependientes la mejora en la velocidad del límite de estabilidad de cada una de las pruebas y la mejora en el porcentaje de relleno del círculo. En este caso, tampoco se obtuvieron datos que permitieran identificar alguna variable que se relacionara con la mejora de los resultados (Tablas 4, 5).

\section{DISCUSIÓN}

La presencia de alteraciones de la personalidad en el vértigo crónico tratado con rehabilitación vestibular afectó a más de la mitad de los pacientes de nuestro estudio, siendo superior al porcentaje obtenido en la mayoría de los trabajos publicados sobre la coexistencia de vértigo y patología psiquiátrica. Es discutible si la persistencia de la sintomatología tras seis meses desde su inicio mantiene la situación de estrés psicológico y, por tanto, favorece la comorbilidad psiquiátrica ó por el contrario, es la presencia de trastornos de personalidad antes

Tabla 3. Regresión logística DHI

\begin{tabular}{|l|c|c|c|c|}
\hline \multicolumn{5}{|c|}{ Modelo Regresión Logística DHI } \\
\hline \multicolumn{1}{|c|}{ Coeficientes } & Estimado & Std.error & z value & $\operatorname{Pr}(>|z|)$ \\
\hline Termino Independiente & 9,83562 & 7135,50273 & 0,001 & 0,9989 \\
\hline Alterado & 0,6951 & 1,16576 & 0,596 & 0,551 \\
\hline $\begin{array}{l}\text { Diagnóstico } \\
\text { V. Central }\end{array}$ & $-16,90036$ & 7135,50183 & $-0,002$ & 0,9981 \\
\hline $\begin{array}{l}\text { Diagnóstico } \\
\text { V. Periférico }\end{array}$ & $-15,52415$ & 7135,50183 & $-0,002$ & 0,9983 \\
\hline Edad & 0,11904 & 0,06274 & 1,897 & 0,0578 \\
\hline Sexo. M & 20,65395 & 2579,1201 & 0,007 & 0,994 \\
\hline
\end{tabular}

Tabla 4. Regresión logística. Límite de estabilidad 1.

\begin{tabular}{|l|c|c|c|c|}
\hline \multicolumn{4}{|c|}{ Modelo Regresión Logística. Límite de estabilidad 1. Velocidad media } \\
\hline \multicolumn{1}{|c|}{ Coeficientes } & Estimado & Std.error & z value & $\operatorname{Pr}(>|z|$ \\
\hline Termino Independiente & 0,371804 & 2,138764 & 0,174 & 0,862 \\
\hline Alterado & $-0,655863$ & 0,600056 & $-1,093$ & 0,274 \\
\hline $\begin{array}{l}\text { Diagnóstico } \\
\text { Central }\end{array}$ & $-0,157278$ & 1,219419 & $-0,129$ & 0,897 \\
\hline $\begin{array}{l}\text { Diagnóstico } \\
\text { Periférico }\end{array}$ & $-0,152881$ & 1,184976 & $-0,129$ & 0,897 \\
\hline Edad & $-0,008962$ & 0,02727 & $-0,329$ & 0,742 \\
\hline Sexo. M & 0,748302 & 0,604155 & 1,239 & 0,215 \\
\hline
\end{tabular}


REHABILITACIÓN VESTIBULAR EN PACIENTES CON VÉRTIGO Y

TRASTORNO DE LA PERSONALIDAD

GUTIÉRREZ-GALLARDO A; SALOM-COVEÑAS C Y PORRAS-ALONSO E C

Tabla 5. Regresión logística. Límite de estabilidad 2.

\begin{tabular}{|l|c|c|c|c|}
\hline \multicolumn{5}{|c|}{ Modelo Regresión Logística. Límite de estabilidad 2. Velocidad media } \\
\hline \multicolumn{1}{|c|}{ Coeficientes } & Estimado & Std.error & z value & $\operatorname{Pr}(>|z|$ \\
\hline Termino Independiente & $-1,48659$ & 2,30074 & $-0,646$ & 0,518 \\
\hline Alterado & $-0,081005$ & 0,063868 & $-1,268$ & 0,205 \\
\hline Diagnóstico central & $-1,74901$ & 1,36199 & $-1,284$ & 0,199 \\
\hline Diagnóstico periférico & $-0,72989$ & 1,30722 & $-0,558$ & 0,577 \\
\hline Edad & $-0,03807$ & 0,02933 & 1,298 & 0,194 \\
\hline Sexo. M & 0,65153 & 0,61775 & 1,055 & 0,292 \\
\hline
\end{tabular}

del inicio de la disfunción vestibular la que influye en el curso clínico del vértigo. Los resultados de Best establecen que, aunque inicialmente en los pacientes con vértigo se asocia un mayor porcentaje de patología psiquiátrica que en controles sanos, esta tendencia va disminuyendo a partir de los seis meses excepto en los pacientes con diagnóstico de migraña vestibular [7]. Asimismo, los trabajos realizados por Savastano sobre características psicológicas de pacientes con síndrome de Ménière concluyen que los síntomas otológicos y las observaciones psicológicas de estos pacientes no son específicas [8]. Es probable que la cronicidad de la sintomatología sea la que favorezca la presencia de las alteraciones de personalidad en estos pacientes. Esta hipótesis se ve reforzada por el mayor número de pacientes con disfunción periférica, en concreto en el síndrome de Ménière y alteraciones de la personalidad y no en pacientes con vértigo central o sin causa orgánica definida.

Con respecto a los subgrupos de los diferentes trastornos de la personalidad definidos por el cuestionario Salamanca y el diagnóstico de disfunción vestibular periférica y central, no se puede establecer ningún rasgo diferenciador a excepción del predominio del grupo $\mathrm{C}$ en pacientes con diagnostico de vértigo periférico. Son los rasgos de personalidad ansiosa y anancástica los predominantes en pacientes con síndrome de Ménière, coincidiendo con los estudios de Eckhardt y Lahmann, diferenciándose de los trastornos asociados a VPPB y neuritis vestibular $[9,10]$. La manifestación clínica de crisis inesperadas, característica de estos pacientes, contribuye a mantener un estado de ansiedad persistente.

Actualmente, existe una evidencia moderada sobre la rehabilitación vestibular en la mejora de los síntomas asociados a la disfunción vestibular periférica y en la percepción de la calidad de vida [11].

El hecho de que el sistema vestibular y el procesamiento de emociones compartan vías neuronales, ha sugerido que el tratamiento de rehabilitación vestibular necesitaría complementarse con otros tratamientos como la terapia cognitiva conductual (CBT) y dialéctica conductual (DBT). La revisión sistemática publicada por Schmid sobre la utilización de la psicoterapia en el vértigo consideró sólo tres ensayos clínicos del total de estudios para analizar la eficacia de la terapia cognitivo-conductual asociada a la rehabilitación vestibular [12]. El resultado obtenido con este planteamiento terapéutico es limitado. Se observa cierta eficacia en el control de algunos síntomas objetivos como los movimientos de provocación del nistagmo o la marcha, así como en la evaluación del DHI, sin evidenciar mejoría relevante en la puntuación de los cuestionarios de valoración de la ansiedad o depresión. Holmberg exploró la utilidad de la terapia combinada en pacientes con vértigo postural fóbico en un ensayo clínico con seguimiento de un año. En este estudio, si hubo un efecto significativamente mayor en el grupo que recibió terapia cognitivo-conductual que en 


\section{REHABILITACIÓN VESTIBULAR EN PACIENTES CON VÉRTIGO Y \\ TRASTORNO DE LA PERSONALIDAD \\ GUTIÉRREZ-GALLARDO A; SALOM-COVEÑAS C Y PORRAS-ALONSO E C}

el grupo en el que sólo se realizó rehabilitación vestibular como tratamiento, que se reflejan en escalas de ansiedad, depresión y en el DHI; con lo que, la terapia cognitivo-conductual tiene un efecto adicional como tratamiento para una población con vértigo postural fóbico. Por lo tanto se sugiere un enfoque multidisciplinario que incluiría: tratamiento médico, terapia cognitivo-conductual y rehabilitación vestibular [13].

\section{CONCLUSIONES}

La mejora en las puntuaciones del DHI y en parámetros objetivos como la velocidad de respuestas en los límites de estabilidad tras la rehabilitación sin otra terapia complementaria en nuestro estudio, sugiere la eficacia del tratamiento en pacientes con sintomatología crónica. Por tanto, el tratamiento rehabilitador como terapia única es útil en pacientes con trastornos de personalidad asociados.

Como conclusión, nuestros resultados sugieren que en pacientes con vestibulopatía periférica crónica, el predominio de rasgos de personalidad ansiosa se relaciona esencialmente con la evolución del cuadro clínico. La rehabilitación vestibular puede ser un tratamiento eficaz en la autopercepción de la discapacidad y en la mejora de los límites de estabilidad en pacientes con disfunción vestibular superior a seis meses, independientemente de su asociación con trastornos de personalidad.

\section{BIBLIOGRAFÍA}

1. Best C, Eckhardt-Henn A, Tschan R, Dieterich M. Psychiatric morbidity and comorbidity in different vestibular vertigo syndromes. Results of a prospective longitudinal study over one year. J Neurol 2009;256:58-65.

2. McDonnell MN, Hillier SL. Vestibular rehabilitation for unilateral peripheral vestibular dysfunction. Cochrane Database Syst Rev 2015;13;1:CD005397.

3. García-Portilla MP, Bascarán MT, Saiz PA, Parellada $\mathrm{M}$, Bousoño $\mathrm{M}$ et al. Banco de instrumentos básicos para la práctica de la psiquiatría clínica. 6 ed. Barcelona: Ars Médica. 2011.

4. Muñoz I, Toribio-Díaz ME, Carod Artal FJ, Peñas-Martínez ML, Ruiz L, Dominguez E et al. Rasgos de personalidad en pacientes migrañosos: studio multicéntrico utilizando el cuestionario de cribado Salamanca. Revista de Neurología. 2013;57(12):529-34.

5. Jacobson GP, Newman CW. The development of the dizziness hándicap inventory. Arch Otolaryngol Head Neck Surg 1990;116:424-27.

6. Pérez N, Garmendia I, Martin E, García-Tapia R. Adaptación cultural de dos cuestionarios de medida de la salud en pacientes con vértigo. Acta Otorrinolaringol Esp 2000;572-80.

7. Best C, Eckhardt-Henn A, Tschan R, Dieterich M. Psychiatric morbidity and comorbidity in different vestibular vertigo syndromes. Results of a prospective longitudinal study over one year. J Neurol 2009;256:58-65.

8. Savastano M, Marioni G, Aita M. Psychological characteristics of patients with Ménière's disease compared with patients with vertigo tinnitus, or hearing loss. Ear, Nose Throat J 2007;86:148-56.

9. Eckhardt-Henn A, Best C, Bense S, Breuer P, Diener G, Tschan R, Dieterich M. Psychiatric comorbidity in different organic vertigo syndromes. J Neurol 2008;255:420-8.

10. Lahmann C, Henningsen P, Dieterich M, Feuerecker R, Cyran CA, Schmid G. Psychiatric comorbidity and psychosocial impairment among patients with vertigo and dizziness. J Neurol Neurosurg Psychiatry 2015;86:302-8.

11. McDonnell MN, Hillier SL. Vestibular rehabilitation for unilateral peripheral vestibular dysfunction. Cochrane Database Syst Rev 2015;13;1:CD005397.

12. Schmid G, Henningsen P, Dieterich M, Sattel $\mathrm{H}$, Lahmann C. Psychotherapy in dizziness:a systematic review. J Neurol Neurosurg Psychiatry 2011;82:601-6.

13. Holmberg J, Karlberg M, Harlacher U, RivanoFischer M, Magnusson M. Treatment of phobic postural vertigo: a controlled study of cognitivebehavioral therapy and self-controlled desensitization. J Neurol 2006;253:500-6. 